\title{
Spontaneous Intradural Cerebral Artery Dissection: Spectrum of Clinical Presentations and Correlation with Angiographic Findings
}

\section{Aminur Rahman ${ }^{1 *}$, Sirintara Pongpech ${ }^{2}$, Pakorn Jiarakongmun ${ }^{2}$, Ekachat Chanthanaphak ${ }^{2}$, Wittawat Takong ${ }^{2}$, Kittiphop Somboonnithiphol ${ }^{2}$ and Thanaboon Worakijthamrongchai ${ }^{2}$}

${ }^{1}$ Assistant Professor, Department of Neurology, Sir Salimullah Medical College Mitford Hospital, Dhaka, Bangladesh

${ }^{2}$ The Division of Interventional Neuroradiology, Department of Radiology,

Ramathibodi Hospital, Faculty of Medicine, Mahidol University, Thailand

*Corresponding Author: Aminur Rahman, Assistant Professor, Department of Neurology, Sir Salimullah Medical College Mitford Hospital, Dhaka, Bangladesh.
Received: August 23, 2021

Published: September 08, 2021

(C) All rights are reserved by Aminur

Rahman., et al.

\begin{abstract}
Aim: Intradural Cerebral Artery dissections are recognized cause of stroke. Aim of this study was to analysis the distribution of spontaneous intradural cerebral artery dissection, angiographic pattern with the symptomatology of admitted patients to our hospital.

Materials and Methods: We analyzed retrospectively collected data of the stroke patients' and carefully evaluated on 4-vessels angiogram in our institute from January 2013 to June 2014. Out of 164 of cerebral dissections in angiographically evidenced we found only 16 patients of intradural dissecting aneurysms that were included in this study. The male-female ratio was $37.5: 62.5$ and the mean age was $47.56 \pm 13.19$ years. According to the angiographic finding depicting the location of the dissection plane in the arterial wall, we categorized to steno-occlusive, aneurysmal, combined pattern. In each dissection pattern, we evaluated presenting symptoms and presence of subarachnoid hemorrhage, infarction, and intracerebral hemorrhage or combined.

Results: The most common symptomatic presentation was headache $(75 \%)$, followed by neck pain (50\%), motor weakness of $\operatorname{limb}(\mathrm{s})$ (43.8\%), loss of consciousness (37.5\%), vertigo (12.5\%), vomiting (12.5) and arm tingling sensation (6.3\%). The most common angiographic pattern was aneurysmal patterns (68.75\%) followed by steno-occlusive (18.75\%) and combined (steno-occlusive and aneurysmal) (12.5\%) patterns. aneurysmal pattern was most frequently related to subarachnoid hemorrhage (SAH) (7/11, $63.63 \%)$ in contrast that steno-occlusive pattern was only related to infarction $(3 / 3,100 \%)$. The most frequent dissection was in the intradural vertebral arteries (IV) and posterior cerebral artery (PCA), presented with SAH 80\% (4/5) and 33.33\% (1/3) respectively followed by infarction and intracerebral hemorrhage (ICH). Infarction was common abnormality in patients with the intradural carotid arteries(IC) 33.33\%(1/3), superior cerebellar artery(SCA) 33.33\%(1/3) and basilar artery(BA) 33.33\% (1/3) each but intracerebral hemorrhage(ICH) was common abnormality in patients with the posterior inferior cerebellar artery(PICA) $50 \%(1 / 2)$.

Conclusion: The most common symptomatic clinical presentations of intradural cerebral artery dissection are headache and neck pain followed by motor weakness of limbs and loss of consciousness. SAH with aneurysmal pattern, in the posterior circulation especially in the vertebral artery is the most frequent diagnosis of in intradural cerebral artery dissection which requires combined analysis of angiographic pattern and clinical presentations of stroke.
\end{abstract}

Keywords: Intradural Cerebral Arteries; Dissection; Aneurysm

\section{Introduction}

Spontaneous intracranial arterial dissection of the carotid and vertebral arteries accounts for only $2 \%$ of stroke [1]. It tends to develop in young healthy people between 25 and 45 years and ac- count for $10-25 \%$ of these patients [2,3]. It usually occurs approximately $90 \%$ of arterial dissections in the posterior circulation due to thin media with fewer elastic fibers [4-6]. Arterial dissections in the posterior circulation usually present with SAH because of their 
elongated subarachnoid course $[7,8]$. In contrast, those of the anterior circulations are generally involved in the supraclinoid segment of the internal carotid artery (ICA) and the middle cerebral artery (MCA) and present with either hemorrhage or ischemia [9,10].

The most symptomatic presentations of cerebral arterial dissection are headache and neck pain. Other clinical presentations vary according to the location of dissection. Dissections have been increasingly diagnosed in recent years due to new development in investigative methods. A diagnosis of cerebral arterial dissection is clinically significant because it can cause severely disabling SAH or ischemic stroke \pm intracerebral hemorrhage (ICH) or combined requiring specific management according to the vessel status demonstrated on cerebral angiography. Different factors are related to underlying structural defect of the arterial wall seem to play an important role in spontaneous arterial dissection [11].

The intradural vertebral artery (IVA) and the cervical ICA are the most frequent involved sites of cerebral arterial dissection [12]. However, there have been few reports on the distribution of intradural cerebral arterial dissection, angiographic pattern and relation to clinical presentations. Clinical symptomatic presentation itself does not always match to the imaging findings of dissection. In this study, we analyzed the distribution of intradural cerebral artery dissection, angiographic pattern as demonstrated on angiograms in 16 patients which were admitted to our hospital.

\section{Materials and Methods}

We analyzed retrospectively collected data of the stroke patients with cerebral angiography in our hospital from January 2013 to June 2014. The diagnosis of arterial dissection was based on the characteristic angiographic findings in association with appropriate clinical history. The cerebral angiography was performed by three neuroradiologists and/or residents under the supervision of the neuroradiologists. To exclude atherosclerotic stenosis or dilation, the characteristic angiographic findings of atherosclerosis included vessel wall irregularity, atheromatous plaque with or without ulceration, tortuousness of all cerebral arteries were carefully evaluated on 4-vessel angiograms. Out of 164 of cerebral dissections in angiographically evidenced we found only 16 patients of intradural dissecting aneurysms that were included in this study. The male-female ratio was 6:10 and the mean age was 47.56 \pm 13.19 years. According to the location and pattern of dissection plane in arterial wall on angiogram we categorized as aneurysmal, steno-occlusive pattern or combined [13-15]. The angiographic findings of the aneurysmal pattern included the ratio of the diameter of dissection and that of normal-looking distal artery is 1.5 or more (Figure 1A.) We categorized the steno-occlusive pattern by the presence of a double lumen, luminal narrowing more than $30 \%$ and gradual tapering ending in total occlusion of the lumen (Figure 1B). When both the steno-occlusive pattern and aneurysmal pattern were found on angiographic analysis, known as combined pattern (Figure 1C). The intradural arteries was based on the observation that the ICA pierced the inner dura immediately proximal to the origin of the ophthalmic artery in the anterior circulation and for the VA, the distinction was made at the point where the artery pierced the dura at the level of foramen magnum. The presence of infarction or hemorrhage such as ICH or SAH was evaluated by reviewing the CT or MRI.

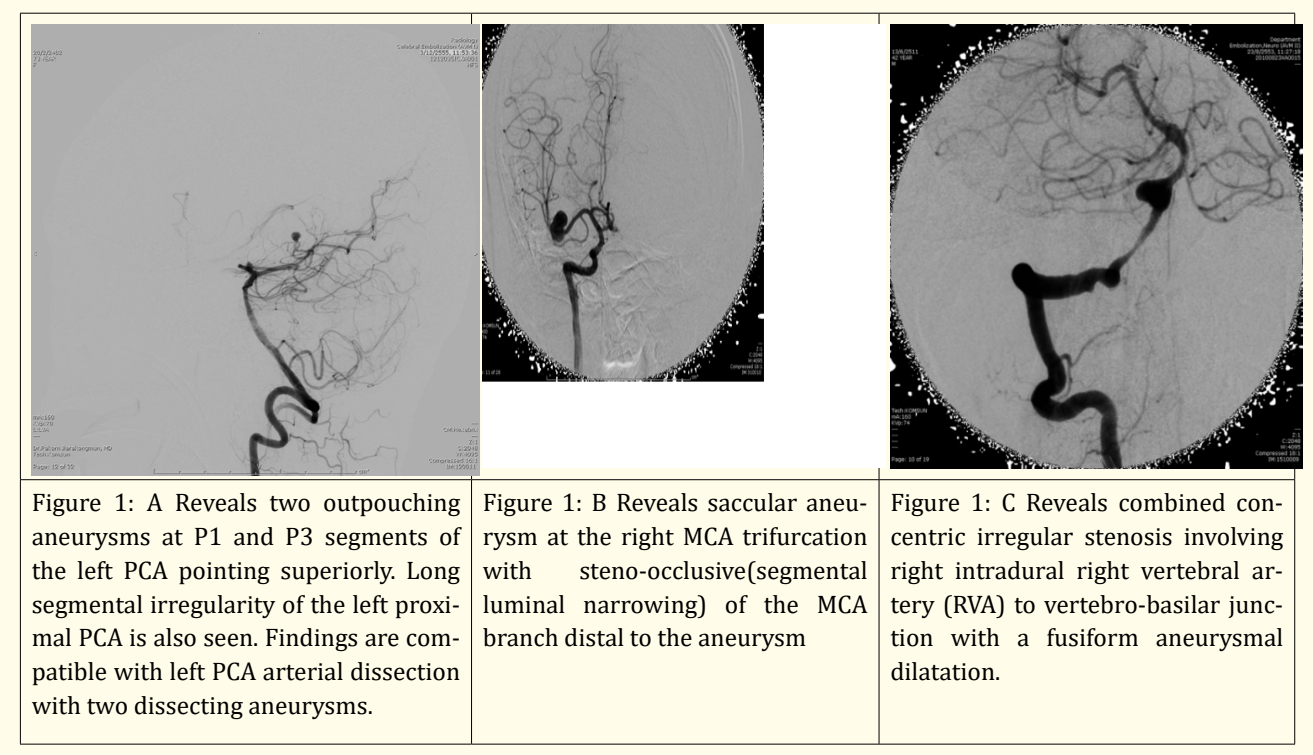

Figure 1

Citation: Aminur Rahman., et al. "Spontaneous Intradural Cerebral Artery Dissection: Spectrum of Clinical Presentations and Correlation with Angiographic Findings". Acta Scientific Nutritional Health 5.10 (2021): 03-08. 


\section{Results}

The most common symptom on presentations were headache (75\%), followed by neck pain (50\%), hemiparesis/hemiplegia (43.8\%), loss of consciousness (LOC)/alteration of consciousness (37.5\%) and vertigo (12.5\%). Other associated symptoms included vomiting and tingling sense of the arm. Main presenting symptoms are listed in table 1 according to the location of dissection.

\begin{tabular}{|c|c|c|c|c|c|c|c|}
\hline Symptoms & IC & PCA & IV & PICA & SCA & BA & Total (\%) \\
\hline Headache & 2 & 4 & 3 & 1 & 1 & 1 & $12(75.0)$ \\
\hline Neck pain & 1 & 3 & 3 & 1 & 0 & 1 & $9(56.25)$ \\
\hline $\begin{array}{c}\text { Hemiparesis/ } \\
\text { hemiplegia }\end{array}$ & 2 & 2 & 1 & 0 & 0 & 1 & $7(43.8)$ \\
\hline $\begin{array}{c}\text { LOC/Alteration of } \\
\text { consciousness }\end{array}$ & 0 & 1 & 1 & 1 & 1 & 2 & $6(37.5)$ \\
\hline Vertigo & 0 & 0 & 0 & 1 & 0 & 1 & $2(12.5)$ \\
\hline Vomiting & 1 & 0 & 1 & 0 & 0 & 0 & $2(12.5)$ \\
\hline $\begin{array}{c}\text { Arm tingling } \\
\text { sensation }\end{array}$ & 0 & 0 & 0 & 0 & 0 & 1 & $1(6.3)$ \\
\hline
\end{tabular}

Table 1: Clinical Presentation Related to the Location of the Dissection.

Abbreviations: LOC, Loss of Consciousness; IC, Intradural Carotid Artery; PCA, Posterior Cerebral Artery; IV, Intradural Vertebral Artery; PICA, Posterior Inferior Cerebellar Artery; SCA, Superior Cerebellar Artery; BA, Basilar Artery.

Angiographic pattern revealed aneurysmal (68.75\%), stenoocclusive (18.75\%), combined (12.5\%) patterns in the order of frequency (Table 2). Lesions were located in the anterior circulation in our 3 patients $(3 / 16,18.75 \%)$ and in the posterior circulation in 13 patients $(13 / 16,81.25 \%)$. The most commonly involved vessels were the intradural vertebral artery (IVA) (5/16, 31.25\%) followed by the intradural carotid artery including its branches $(3 / 16$, $18.75 \%)$, and the posterior cerebral artery (PCA) $(3 / 16,18.75 \%)$ (Table 2). In the dissection of IVA, the most common angiographic pattern was aneurysmal pattern combined pattern $(4 / 11,36.36 \%)$ followed by steno-occlusive pattern $(1 / 11,9.1 \%)$.

\begin{tabular}{|c|c|c|c|c|c|c|c|}
\hline & IC & PCA & IVA & PICA & SCA & BA & Total \\
\hline Aneurysm & 0 & 3 & 4 & 1 & 1 & 2 & $11(68.75 \%)$ \\
\hline Steno-occlusive & 2 & 0 & 1 & 0 & 0 & 0 & $3(18.75 \%)$ \\
\hline Combined & 1 & 0 & 0 & 1 & 0 & 0 & $2(12.5 \%$ \\
\hline Total & 3 & 3 & 5 & 2 & 1 & 2 & $16(100 \%)$ \\
\hline
\end{tabular}

Table 2: Angiographic Patterns Related to the Location of the Dissection.
Analysis of MR and/or CT revealed SAH (50\%), infarction (18.75\%), ICH (12.5\%), combined (6.25\%) and none (12.5\%) (Table 3). Aneurysmal pattern of cerebral artery dissection was most commonly related to SAH $(7 / 11,66.63 \%)$. Steno-occlusive pattern was most frequently related to infarction $(3 / 3,100 \%)$. In patients with combined pattern of both steno-occlusion and aneurysm, SAH was the most common presenting pattern on abnormal CT/MR findings $(1 / 2,50 \%)$ (Table 3$)$.

\begin{tabular}{|c|c|c|c|c|}
\hline & Stenocclusive & Aneurysm & Combined & Total \\
\hline SAH & 0 & 7 & 1 & $8(50 \%)$ \\
\hline Infarction & 3 & 0 & 0 & $3(18.75 \%)$ \\
\hline ICH & 0 & 1 & 1 & $2(12.5 \%)$ \\
\hline Combined & 0 & 1 & 0 & $1(6.25 \%)$ \\
\hline None & 0 & 2 & 0 & $2(12.5 \%)$ \\
\hline Total & 3 & 11 & 2 & $16(100 \%)$ \\
\hline
\end{tabular}

Table 3: Clinical Findings Related to Angiographic Pattern. SAH, Subarachnoid Hemorrhage; ICH, Intracerebral Hemorrhage.

The most frequent abnormality detected on CT or MRI in patients with dissection of the IV was SAH (4/8). Infarction was the common abnormality on CT or MRI in patients with subsequent IC $(2 / 3)$ and BA (1/3). ICH was the most common abnormality on CT or MRI in patients with PICA (1/1). As expected, no infarction was detected in patients with dissection of IV. One (1/2) patient in IV group had no SAH and infarction at the same time (Table 4).

\begin{tabular}{|c|c|c|c|c|c|c|c|}
\hline & IC & PCA & IV & PICA & SCA & BA & Total \\
\hline SAH & 0 & 1 & 4 & 1 & 1 & 1 & 8 \\
\hline ICH & 1 & 0 & 0 & 1 & 0 & 0 & 2 \\
\hline Infarction & 2 & 0 & 0 & 0 & 0 & 1 & 3 \\
\hline Combined & 0 & 1 & 0 & 0 & 0 & 0 & 1 \\
\hline None & 0 & 1 & 1 & 0 & 0 & 0 & 2 \\
\hline Total & 3 & 3 & 5 & 2 & 1 & 2 & 16 \\
\hline
\end{tabular}

Table 4: Clinical Findings Related to the Location of Dissection.

\section{Discussion}

Spontaneous dissections of the carotid and vertebral arteries affect all age groups, including children, but there is a distinct peak in the fifth decade of life $[16,17]$. Although there is no overall sexbased predilection, female are on average about five years younger than male at the time of the dissection.16 In our study the mean age of the population was $47.56 \pm 13.19$ years is similar to observa- 
tions in other series $[18,19]$ and male-female ratio was 37.5: 62.5 .

Headache and neck pain are the common symptoms associated with spontaneous artery dissection [20,21]. In our study showed that the most common symptomatic presentation was headache (75\%) followed by neck pain (50\%), motor weakness of limb(s) (43.8\%), loss of consciousness (37.5\%), vertigo (12.5\%), vomiting (12.5) and arm tingling sensation (6.3\%) of spontaneous artery dissection. The study also revealed that most common symptomatic presentation were headache (83.33\%) and neck pain (88.88\%) in the posterior circulation especially IVA and PCA whereas hemiparesis/hemiplegia (28.57\%) was the common symptom than a headache(16.66\%) in the intradural ICA.

Several population-based studies showed that the annual incidence of spontaneous carotid artery dissection is 2.5 to 3 per 100,000 , while the annual incidence of spontaneous vertebral artery dissection is 1 to 1.5 per 100,000 [22,23]. Dissecting aneurysms of the vertebral artery constitute $4 \%$ of all cerebral aneurysms, and are hence a relatively rare but important cause of subarachnoid hemorrhage [23]. In our study, we found that the IVA was more frequently involved $(31.25 \%)$ than the intradural ICA $(18.75 \%)$. This result was quite different previously reported data from the western country due to more frequent incidence of small vessel disease in Asian population. In addition, histologically the IV arterial wall has thinner tunica media is and no external elastic lamina [24]. That is why the predominance of spontaneous vertebrobasilar dissection without any etiology [25].

The angiographic pattern related to location of arterial dissection was variable. The steno-occlusion pattern was more common in the intradural carotid artery. The aneurysmal and combined pattern was more common in the IVA and presented with SAH and ICH $[10,26]$. In contrast that steno-occlusive pattern presented with ischemic stroke. Although such dissecting aneurysm needs to be differentiated from atherosclerotic vessel wall change, underlying atherosclerotic changes might contribute to the cause of dissection [27]. Our Aneurysmal pattern of cerebral artery dissection was most commonly related to SAH $(7 / 11,66.63 \%)$ and $\mathrm{ICH}(1 / 2,50 \%)$ mostly in the IVA. Steno-occlusive pattern was most frequently related to infarction $(3 / 3,100 \%)$. In patients with combined pattern of both steno-occlusion and aneurysm, SAH was the most common presenting pattern on abnormal CT/MR findings $(1 / 2,50 \%)$. Our result was consistent with the previous studies.
There have been few reports on the cerebral artery dissection whose distribution was analyzed in the concept of diameter of the vessels. We supposed that the diameter of cerebral artery contributed to decide the type of dissection. A subintimal dissection tends to result in stenosis of the arterial lumen that may cause infarction due to flow disturbance. On the other hand, a subadventitial dissection may cause aneurysmal dilation of the artery, which can cause SAH $[28,29]$. Several studies reported that arterial stenosis with dilatation occurred predominantly in SAH cases, whereas stenosis without dilatation arose mainly in ischemic cases [29,30]. Because the ICA has the larger diameter than the intradural VA and PCA, they have thicker media which tends to maintain the adventitia of carotid artery. The IC presented frequently with ischemia as in our study because they might have more subintimal than subadventitial type dissections. So in our study showed that, dissection involved to the IC group (18.75\%) most likely steno-occlusive pattern due to thicker media of IC.

Our study also revealed that dissection involved to the IV $(68.75 \%)$ was the most aneurysmal pattern and was presented with SAH. The main trunk of VA has a long part in the subarachnoid space and the intradural segment of the VA has a thinner media and adventitia, and dissections involving the segment are more likely to extend subadventitially producing SAH [31].

Dissection has variable clinical outcome depending on location and angiographic pattern. Intradural arterial dissections result in more occurrence of SAH caused by aneurysmal pattern, especially in the intradural vertebral artery whereas ischemia caused by steno-occlusive pattern was presented in the IC.

\section{Conclusion}

The most common symptomatic clinical presentations of intradural cerebral artery dissection are headache and neck pain followed by motor weakness of limbs and loss of consciousness. SAH with aneurysmal pattern, in the posterior circulation especially in the vertebral artery is the most frequent diagnosis of in intradural cerebral artery dissection which requires combined analysis of angiographic pattern and clinical presentations of stroke. Careful analysis and follow-up based on the angiographic finding and clinical presentation is mandatory because some patients presented with SAH in steno occlusive pattern or infarction in aneurismal pattern. 


\section{Acknowledgement}

We are thankful to the resident doctors, The Division of Interventional Neuroradiology, Department of Radiology, Ramathibodi Hospital, Faculty of Medicine, Mahidol University for their help in data collection. We also acknowledge the all the staffs in the Department of Radiology, Ramathibodi Hospital, for their assistance.

\section{Statement of Ethics}

The authors had the approval from the Faculty of Medicine, Mahidol University, Ethical Committee.

\section{Conflict of Interest Statement}

This research was done during training period of Interventional Neuroradiology, Department of Radiology, Ramathibodi Hospital, Faculty of Medicine, Mahidol University. The authors have no conflicts of interest to disclose.

\section{Funding Sources}

The authors did not receive funding from any source.

\section{Bibliography}

1. Kim YK and Schulman S. "Cervical artery dissection: pathology, epidemiology and management". Thrombosis Research 123.6 (2009): 810-821.

2. Ducrocq X., et al. "Accidents vasculaarescérébrauxischémiques du sujetjeune". Étude prospective de 296 patients âgés de 16 à 45 ans. Review on Neurology 155 (1999): 575-582.

3. Hart RG and Easton JD. "Dissections". Stroke 16 (1985): 925927.

4. Suzuki K., et al. "Anterior cerebral artery dissection presenting subarachnoid hemorrhage and cerebral infarction". Journal of Nippon Medical School 79.2 (2012): 153-158.

5. Yamaura A., et al. "Clinical picture of intracranial non-traumatic dissecting aneurysm". Neuropathology 20.1 (2000): 85-90.

6. Endo S., et al. "A pathological study of intracranial posterior circulation dissecting aneurysms with subarachnoid hemorrhage: report of three autopsied cases and review of the literature". Neurosurgery 33 (1993): 732-738.

7. Amagasa M., et al. "Posttraumatic dissecting aneurysm of the anterior cerebral artery: case report". Neurosurgery 23.2 (1988): 221-225.
8. Huang YC., et al. "Cervicocranial arterial dissection: experience of 73 patients in a single center". Surgical Neurology 72 (2009): S20-27; discussion S27.

9. Ohkuma H., et al. "Neuroradiologic and clinical features of arterial dissection of the anterior cerebral artery". AJNR American Journal of Neuroradiology 24.4 (2003): 691-699.

10. Suzuki I., et al. "Nontraumatic arterial dissection of the anterior cerebral artery: six cases report". No To Shinkei. 57.6 (2005): 509-515.

11. Krings $\mathrm{T}$ and Choi IS. "The many faces of intracranial arterial dissections". Interventional Neuroradiology 16 (2010): 151160.

12. Shin JH., et al. "Vertebral artery dissection: spectrum of imaging findings with emphasis on angiography and correlation with clinical presentation". Radiographics 20 (2000): 16871696.

13. Touzé E., et al. "Aneurysmal forms of cervical artery dissection: associated factors and outcome". Stroke 32 (2001): 418-423.

14. Hart RG and Easton JD. "Dissections of cervical and cerebral arteries". Neurology Clinics 1 (1983): 155-182.

15. Kwak JH., et al. "Cerebral Artery Dissection: Spectrum of Clinical Presentations Related to Angiographic Findings". Neurointervention 6.2 (2011): 78-83.

16. Schievink WI., et al. "Recurrent spontaneous cervical-artery dissection". The New England Journal of Medicine 330 (1994): 393-397.

17. Schievink WI., et al. "Spontaneous dissections of cervicocephalic arteries in childhood and adolescence". Neurology 44 (1994): 1607-1612.

18. Chaves C., et al. "Spontaneous intracranial internal carotid artery dissection". Archives of Neurology 59 (2002): 977-9811.

19. Kocaeli H., et al. "Spontaneous intradural vertebral artery dissection: a single center experience and review of literature". Skull Base 19 (2009): 209-218.

20. Li S., et al. "Prognosis of intracranial dissection relates to site and presenting features". Journal of Clinical Neuroscience 18 (2011): 789-793.

21. Silbert PL., et al. "Headache and neck pain in spontaneous internal carotid and vertebral artery dissections". Neurology 45 (1995): 1517-1522. 
22. Bogousslavsky J and Regli F. "Ischemic stroke in adults younger than 30 years of age. Cause and prognosis". Archives of Neurology 44 (1987): 479-482.

23. Lucas C., et al. "Stroke patterns of internal carotid artery dissection in 40 patients". Stroke 29 (1998): 2646-2648.

24. Strong KC. "A study of the structure of the media of the distributing arteries by the method of microdissection". The Anatomical Record 72 (1938): 151-167.

25. Kocaeli H., et al. "Spontaneous intradural vertebral artery dissection: a single-center experience and review of the literature". Skull Base 19 (2009): 209-218.

26. Mizutani T., et al. "Proposed classification of nonatherosclerotic cerebral fusiform and dissecting aneurysms". Neurosurgery 45 (1999): 253-259.

27. In HS., et al. "Intracranial stenting in patients with atherosclerotic stenosis associated with various aneurysms in the same diseased arterial segment". AJNR American Journal of Neuroradiology 31 (2010): 1895-1898.

28. Yonas H., et al. "Dissecting intracranial aneurysms". Surgical Neurology 8 (1977): 407-415.

29. Yamada SM., et al. "Dissecting aneurysm of the anterior cerebral artery with severe subarachnoid hemorrhage during treatment for cerebral infarction". Internal Medicine 42 (2003): 433-435.

30. Ohkuma H., et al. "Dissecting aneurysms of intracranial carotid circulation". Stroke 33 (2002): 941-947.

31. Mizutani T. "Natural course of intracranial arterial dissections". Journal of Neurosurgery 114 (2011): 1037-1044.

\section{Volume 4 Issue 10 October 2021}

\section{(C) All rights are reserved by Aminur Rahman., et al.}

